\title{
Viewpoint
}

\section{Rotations of the Hoyle State in Carbon-12}

\author{
Hans O. U. Fynbo \\ Department of Physics and Astronomy, Aarhus University, DK-8000 Aarhus C, Denmark
}

\author{
Martin Freer \\ School of Physics and Astronomy, University of Birmingham, Birmingham B15 2TT, \\ United Kingdom
}

Published November 14, 2011

\begin{abstract}
New scattering experiments provide evidence for the long-suspected existence of an excited rotational state in carbon-12, related to a state that's crucial in stellar fusion reactions.
\end{abstract}

Subject Areas: Astrophysics, Nuclear Physics

\author{
A Viewpoint on: \\ Candidate for the $2+$ excited Hoyle state at Ex $10 \mathrm{MeV}$ in $12 \mathrm{C}$ \\ M. Itoh, H. Akimune, M. Fujiwara, U. Garg, N. Hashimoto, T. Kawabata, K. Kawase, S. Kishi, T. Murakami, K. \\ Nakanishi, Y. Nakatsugawa, B. K. Nayak, S. Okumura, H. Sakaguchi, H. Takeda, S. Terashima, M. Uchida, Y. \\ Yasuda, M. Yosoi, and J. Zenihiro \\ Physical Review C 84, 0543082011 - Published November 14, 2011
}

The astrophysical synthesis of carbon and heavier elements, essential to the existence of planets and life, depends on a quirk of nuclear physics. Fusion of two helium-4 nuclei in the hot cores of stars easily creates beryllium-8, and fusion of that nucleus with another helium-4 can produce carbon-12. However, beryllium8 has a very short lifetime against alpha decay, implying that it should almost always break up rather than become the seed for carbon. To get around this roadblock, the astrophysicist Fred Hoyle suggested in 1953 that carbon-12 must have an excited state with approximately the same energy as the combination beryllium- 8 plus helium-4[1]. This resonance markedly increases the cross section for the fusion reaction producing carbon-12, allowing stellar nucleosynthesis to proceed to still heavier elements. The excited state, with an energy of 7.6 million electron volts $(\mathrm{MeV})$, total angular momentum $J=0$, and positive parity, indeed exists, and is often referred to as the "Hoyle state."

The detailed structure of the Hoyle state is even now a matter of some doubt, creating uncertainty in the carbon12 production cross section that is needed for stellar models that calculate the abundance of carbon and other elements. Only three years after Hoyle made his proposal, nuclear physicist Haruhiko Morinaga conjectured that the Hoyle state should be highly nonspherical, and that as a result the $J=0$ state should be accompanied by a band of rotational excited states with angular momentum $J=2,4$, and so on 2. (Similar structures are seen in beryllium-8, oxygen-16, neon-20, and magnesium24.) Numerous experiments over the past 50 years have failed to locate these additional states in carbon-12, but

DOI: 10.1103/Physics.4.94

URL: http://link .aps .org/doi/10.1103/Physics .4.94
Masatoshi Itoh of Tohoku University, Japan, and colleagues, writing in Physical Review $C[3$, now present experimental evidence for the existence of a $J=2$ state close to where it was originally predicted by Morinaga, about $2 \mathrm{MeV}$ above the Hoyle state. Similar results from Freer et al. 4 and Zimmerman et al. 5 . make it plausible that the $J=2$ state has finally been found, and there are even hints of the next rotational state, with $J=4[6]$.

The significance of this state lies not only in its role in the synthesis of carbon, but also in its characteristic structure. Ikeda et al. 7 postulated that close to an alpha-decay threshold, i.e., in an excited state with internal energy sufficient for an alpha particle to escape the nucleus, states including a preformed alpha particle (or helium-4 nucleus) should appear. Such states, called cluster states, were predicted remarkably early in the history of nuclear physics, even before the 1932 discovery of the neutron by Chadwick. A few years later, Hafstad and Teller [8] provided a detailed characterization of the arrangement of the clusters. In the case of carbon-12, the Hoyle state resides just above the threshold for decay into beryllium-8 plus an alpha; in turn, beryllium-8's ground state lies $92 \mathrm{keV}$ above the 2-alpha decay threshold. Ikeda's hypothesis implies, then, that the Hoyle state should have a 3 -alpha cluster structure. The precise nature of the cluster's structure has remained elusive, but the recent measurements provide some insight. Further studies may make it possible to determine whether the cluster has a fairly rigid crystal-like structure, with the alphas arranged in chain, triangle, or other structure, or a gaslike structure in which they move around more freely (Fig. 1).

(c) 2011 American Physical Society 


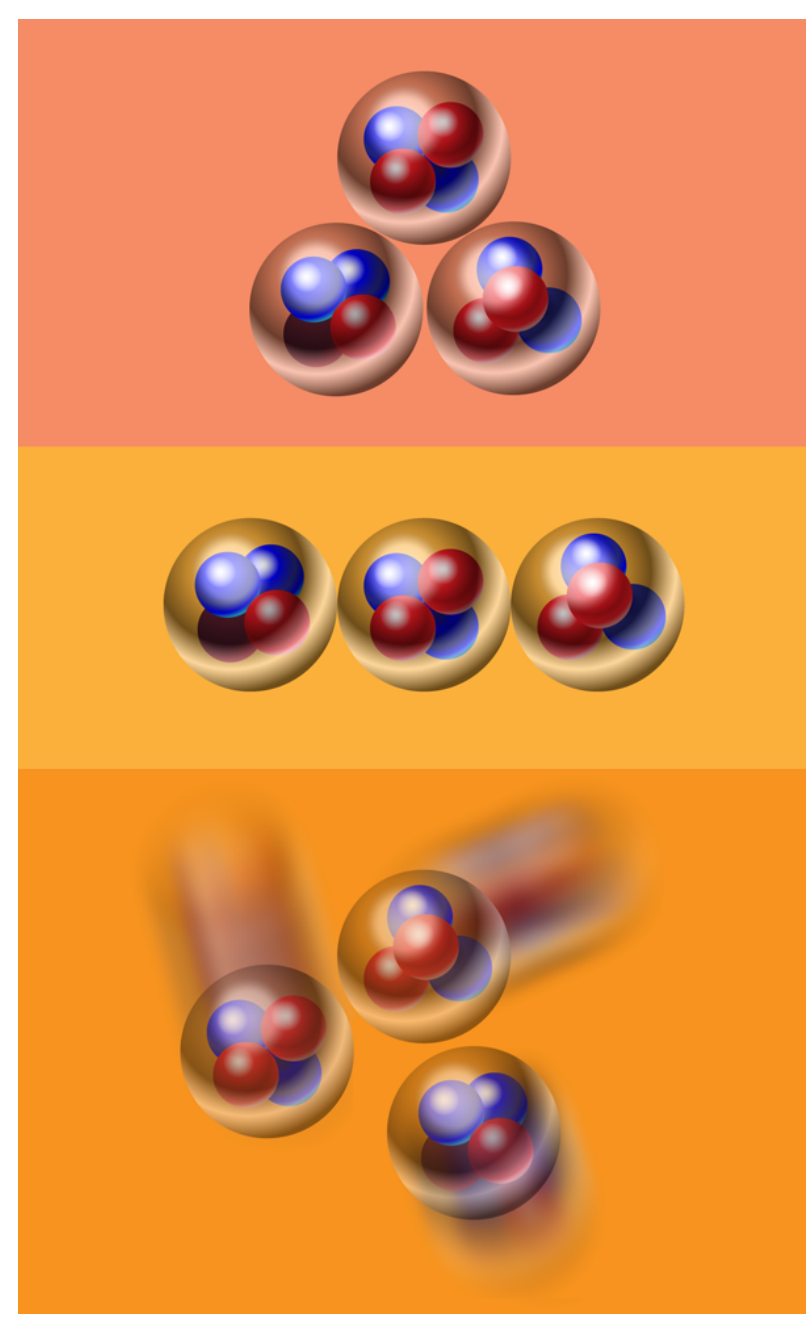

FIG. 1: Excitations of the Hoyle state in carbon-12 are thought to exist as so-called cluster states in which the six protons and six neutrons aggregate into three alpha particles. Recent experiments provide new evidence for the existence of these states, and may shed light on whether the alpha particles arrange themselves in a triangle, a chain, or other structure, or move more or less freely around each other. (APS/Alan Stonebraker)

The difficulty in identifying the $2^{+}(J=2$, with positive parity) excitation of the Hoyle state in carbon- 12 stems from the fact that all states above $7.3 \mathrm{MeV}$ are unstable with respect to disintegration into three alpha particles. They are, therefore, resonances with short lifetimes and correspondingly large uncertainties, up to several $\mathrm{MeV}$, in their energies. Close to Morinaga's $2^{+}$rotational state there are also other known resonances with $J=3$ and $J=0$, and in most experiments these other states dominate the measured spectra. Itoh et al. solve this by letting a high-energy beam of alpha particles scatter from a carbon-12 target and measuring very accurately the energy and deflection angle of the scattered alphas. For the smallest angles, scattering from the $J=0$ dominates; for somewhat larger angles, the $J=2$ state dominates, and so on. Each value of $J$ leads to a characteristic intensity pattern for the scattered particles. In this way, Itoh et al. are able to decompose the scattered particle distribution into components corresponding to different angular momentum components in the carbon12 nucleus. In their results, they see a small contribution from a $J=2$ state beneath dominant contributions from the $J=0$ and $J=3$ states. Freer et al. 4 and Zimmerman et al. 5 use a similar technique to provide selectivity for $J=2$ over other angular momentum states, but they use beams of protons instead of alphas.

It is tempting to pronounce the question closed. However, other experiments find no evidence for the proposed $2^{+}$state. For example, high-precision measurements of the beta-plus decay of nitrogen-12 and the beta-minus decay of boron-12, both of which produce carbon-12, do not show any significant strength close to the region where the $J=2$ state found by Itoh et al. is observed [9]. The situation therefore remains somewhat confusing: Does the $J=2$ state exist or does it not, and if it does exist, why is it so weakly populated in many of the reactions? An answer to at least the second question, in the case of the inelastic scattering measurements, may be that the pronounced cluster structure of the new $2^{+}$state has a small overlap with the carbon-12 ground state, leading to a small likelihood for excitation to that state [10]. A similar explanation might be advanced for the lack of observation in the beta-decay measurements, where the small structural overlap between parent and daughter wave functions leads to a small nuclear matrix element for the decay.

Although the measurements by Itoh et al., coupled with those published previously, provide a significant step toward solving the 50 -year-old puzzle of the $J=2$ state, there is still a need for further investigation. On the theoretical side, the long term goal will be to develop a consistent description combining state-of-the-art structure calculations with models for the nuclear reactions or decays used in the experiments. On the experimental side, new precise measurements will be needed, possibly including new approaches for enhancing the selectivity between different angular momentum states.

\section{References}

[1] F. Hoyle, D. N. F Dunbar, W. A. Wenzel, W. Whaling, Phys. Rev. 92, 1095c (1953) in Minutes of the New Mexico Meeting Held at Alberquerque, September 2-5.

[2] H. Morinaga, Phys. Rev. 101, 254 (1956).

[3] M. Itoh et al., Phys. Rev. C 84, 054308 (2011).

[4] M. Freer et al., Phys. Rev. C 80, 041303 (2009).

[5] W. R. Zimmerman, N. E. Destefano, M. Freer, M. Gai, and F. D. Smith, Phys. Rev. C 84, 027304 (2011).

[6] M. Freer et al., Phys. Rev. C 83, 034314 (2011).

[7] K. Ikeda et al., Prog. Theor. Phys. Suppl., Extra Number, 464(1968).

[8] L. R. Hafstad and E. Teller, Phys. Rev. 54, 681 (1938).

[9] S. Hyldegaard et al., Phys. Rev. C 81, 024303 (2010). 
[10] Dao T. Khoa, Do Cong Cuong, and Yoshiko Kanada-En'yo, Phys. Lett. B 695, 469 (2011).

\section{About the Authors}

\section{Hans O. U. Fynbo}

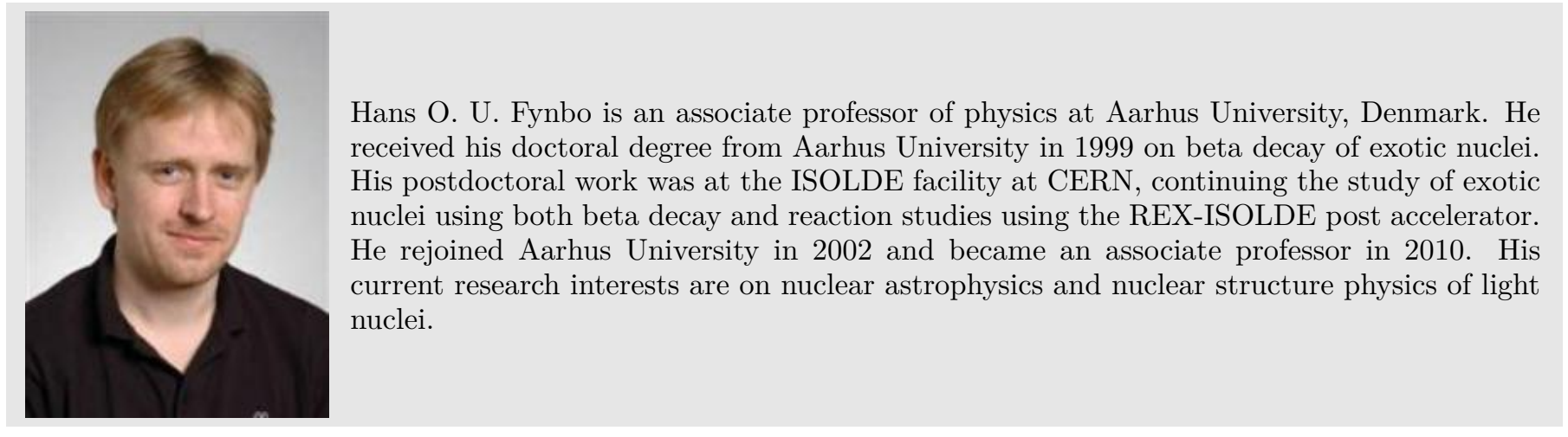

\section{Martin Freer}

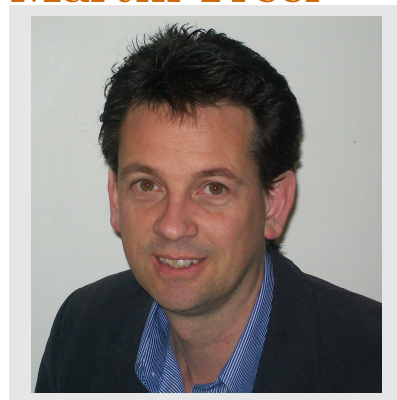

Professor Martin Freer is director of the Birmingham Centre for Nuclear Education and Research. His research includes the characterization of alpha-cluster states and nuclear molecules, the latter being a set of states whereby neutrons are exchanged between alpha particles, analogous to atomic molecules. He was awarded the 2010 Rutherford medal for his contribution to the study of nuclear clustering. 